\title{
Developing Expert System with Soft Systems Concept
}

\author{
Chuk Yau and Abdul Sattar \\ School of Computing and Information Technology \\ Griffith University \\ Nathan, Brisbane (Australia) 4111 \\ \{chuk,sattar\}@cit.gu.edu.au
}

\begin{abstract}
The traditional approaches used for the development of expert systems are mainly based on the interaction between the knowledge engineer and the domain expert. However, the role of user in the development process has been undermined. The human factors involved in the user/expert-system interaction are fundamental to the development of expert system and must be taken into account. This paper adopts the soft systems concept to represent the expert system development as a soft system which allows us to consider all relevant facets of the environment. Based on the soft systems concept, a new methodology for establishing the expert system development system (ESDS) is introduced.
\end{abstract}

\section{Introduction}

An expert system is a software package which emulates the decision-making ability of a human expert in a specific domain. For example, building computer programs for diagnosing a patient based on a given set of symptoms and advising him of an appropriate treatment [2], the design and diagnosis of faults in electronic circuits [6], the characterisation of oil and gas fields with an assessment of their hydrocarbon potential [4] or configuration of computer systems[8]. The underlying knowledge representation scheme for most of these expert systems is production system sometimes called a rulebased approach. In general, the productions systems are computationally as powerful as other computable functions such as Turing machine, predicate logic, lambda calculus etc. However, it has been argued that if ... then ... kind of rules are fundamental to capture the human expert knowledge [7].

It is not difficult to see the commercial values of the expert systems developed during last 20 years. For example, Prospector [11] was successfully used in mineral deposit exploration, and XCON [8] has saved DEC from a lot of computer configuration work since 1983 .

Among many proposed methods for building expert systems, the prototyping approach has been identified as an appropriate procedure. Because the development of expert systems involves understanding of human knowledge which can hardly be completed without obtaining feedback from all relevant parties in the problem environment.

While developing knowledge-based systems, we must address the two fundamental problems: adequately representing and understanding the expert knowledge; and dealing with the dynamic (soft) nature of knowledge. The traditional approaches mainly based on the interaction between the knowledge engineer and the domain expert. However, the role of user in the development process has been undermined. For example, how the relationship between user and other agents in the environment affects the process of building human understandable programs that exhibits intelligence. We argue that the human factors involved in the user/expert-system interaction are fundamental to the development of expert system and must be taken into account. 


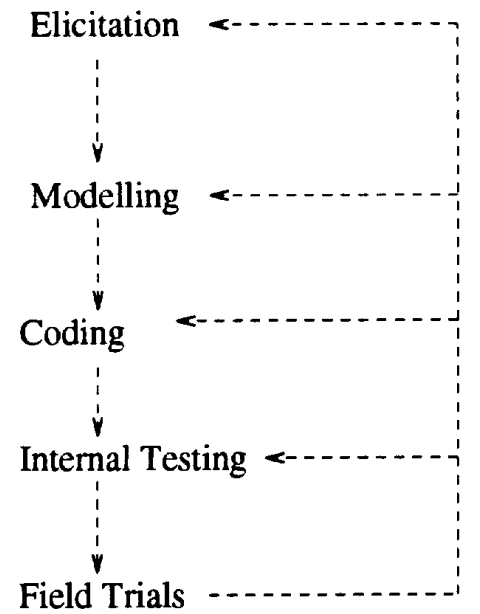

Figure 1: Knowledge-Based System Development Process

\section{Expert System Develop- ment}

In the expert system development (see Figure 1), between the steps of knowledge elicitation and modelling, there is a feedback loop representing the adjustment of inconsistencies due to misunderstanding between the domain experts and the knowledge engineer. There are also back loop opportunities for other steps for improving the model and the codes. The field trials review the prototype system and provide the domain experts and knowledge engineers with advice to improve the knowledge base.

The fundamental goal of knowledge based approach is to develop an expert program that is capable of solving problems at least as good as human experts.

\subsection{Components of an Expert Sys- tem}

A typical expert system consists of four components: knowledge base, user interface, working memory and inference engine.

The user interface acts as a pre-processing system that performs syntactic and semantic analysis on user input. The relevant information is extracted and stored in the working memory.
The user interface corresponds to the sensory and responding system in the human cognition process. Ideally, it should involve understanding natural language and even machine perception. However, the research on natural language understanding and computer perception has not progressed enough to achieve such goals. Most expert systems today use a higher level language to interact with users.

The knowledge base of an expert system resembles the permanent memory of an human expert. Domain-specific knowledge is placed there. This knowledge base is used by the inference engine to derive conclusions or solutions to the problems. The inference engine resembles the thinking part of human cognition process. It mainly directs the flows of information and performs reasoning based on the working memory, the knowledge base and the external environment. The inference engine interprets the facts of the working memory, applies the knowledge in the knowledge base to construct plans and to solve problems.

In the expert system development, our focus is the three main issues: knowledge acquisition, knowledge representation, and utilisation of the knowledge.

\subsection{Knowledge Acquisition}

Knowledge acquisition is a process which aims at extracting knowledge, experience and problemsolving procedures from one or more domain experts. There are many general and specific knowledge acquisition approaches available. A general knowledge acquisition approach consists of five stages as shown in Figure 1.

The first stage, elicitation, aims at extracting domain experts' knowledge about the problem under consideration. Modelling is the process for transforming the findings of the first stage into more formal knowledge representations. There is a feedback loop between these two stages that allows to make corrections of inconsistencies which might have caused by misunderstanding between the expert and the knowledge engineer. Internal testing is verification process to ensure the correctness of the development before field trials can be proceeded. The field trial requires the involvement of all relevant parties to validate the developed product. 
The efficiency and effectiveness of the process of knowledge acquisition are affected by the following $[5]$ :

- Experts may not be able to express their knowledge and experience in a structured way.

- Experts may not be aware of the significance of the knowledge they have used.

- Even experts can express their knowledge, it may be irrelevant, incomplete, incorrect and not understandable

Normally, domain expert are not necessary to familiar with computers. In order to acquire their knowledge to meet the requirements of the development of expert systems, knowledge engineers are needed to bridge the gap between a computer system and domain experts.

There are many methods used in knowledge acquisition and could be classified into 2 groups [10], namely direct and indirect methods.

- Direct Approach: The knowledge engineer asks the expert to report on knowledge that he can directly articulate. The following methods are commonly used as direct approach.

- Interview

- Questionnaires

- Observation of the task performance

- Protocol analysis

- Interruption analysis

- Drawing closed curves

- Inference flow analysis

- Indirect Approach: The expert are not asked to express their knowledge directly but are asked some questions. By asking these questions, other behaviour is collected. Some examples of indirect approach are as following:

- Multidimensional scaling

- Johnson hierarchical clustering

- Ordered tree from recall

- Repertory grid analysis
The choice of the methods to be used depends on the domain field, the domain expert and the real situation. It is not uncommon that more than one method are used to ensure full understanding of the real life situation. However, interviewing the domain expert is frequently included as part of a knowledge acquisition process.

Normally the knowledge acquisition is an iterative process which allows formulated knowledge to be verified and validated from time to time. In brief, the knowledge engineer extract knowledge from domain expert and then organise it in the form that can be utilised by an expert system. If the expert system can not provide satisfactory outcome, the domain expert will be consulted again for improvement. The amount of effort expended and the number of iterations required depend on the size of the system to be built, the depth and breath of the tasks to be supported, and the quality of the knowledge as it is acquired [9].

\subsection{Knowledge Representation and Utilisation}

In addition to knowledge acquisition, there are two more key issues we have to consider while building an expert system: knowledge representation and utilisation of the acquired knowledge. A number knowledge representation schemes available to the knowledge engineer such as production rules, semantic network, frames, logic, and objected-oriented extensions of existing knowledge representation languages. Knowledge utilisation can also be viewed as knowledge maintenance. In another words, it requires an up to date knowledge base which can be used sensibly and consistently.

The knowledge that human experts possess is dynamic. Normally, expert systems are built incrementally in the sense that knowledge can be added and revised from time to time. In conventional problem solving approaches such as operational research techniques, knowledge is embedded in the algorithm that may deal with the problem with a specific problem environment. Changes in the knowledge used are difficult to make and usually result in re-designing the whole algorithm. On the contrary, the representation of knowledge is usually separated clearly from the inference mechanism or reason- 
ing system in an expert system, making it more convenient to add or remove knowledge without altering the inference mechanism.

\section{The Soft System Concept}

Real life problems lie between two extreme ends namely soft and hard. According to Checkland [3], a soft problem is:

\begin{abstract}
"a problem, usually a real-world problem, which cannot be formulated as a search for an efficient means of achieving a defined end; a problem in which ends, goals, purpose are themselves problematic."
\end{abstract}

Thus it is impractical to formulate definite procedures to solve soft problems. Checkland proposes a soft system methodology (SSM) [3] for handling this particular type of real-world problems. The soft systems concept provides a thinking framework to represent the problem environment as a human activity system for visualising internal and external environment factors as an integrated entity. This concept also allows recognition of the proper place and function of all associated elements. The systems within which the different parties must be involved are necessarily complex. The soft systems concept is expected to help to dissolve some of the complexity, and it can also contribute to clarifying the complex problems and thereby operate within the perceived environment. The construction of a human activity system should consider the following elements:

- objective

- components

- connectivity

- transformation

- subsystems and wider systems

- measures of performance

\section{Soft Systems Approach to Expert System Develop- ment}

The main problem of expert system development is due to the dynamic nature of knowledge. Firstly, understanding and expressing expert knowledge is always not an easy task. Secondly, the knowledge may be changed from time to time which is subjected to additional constraints that may not be known at the initial stage of the development. This is particularly common to business management problems. The main idea of adopting the soft systems concept is not only to improve the development of expert system but also try to help the associated parties to clarify their problem situation. Through the soft systems concept, we identify some weaknesses of the traditional knowledge acquisition techniques.

The first problem is related to the human resource involvement. Although all knowledge acquisition approaches indicate that the development of expert system requires the involvement of the knowledge engineer, domain expert and users, most knowledge acquisition methods focus on the interaction between the knowledge engineer and the domain expert. The lack of contributions from the users would reduce the usability of the knowledge based systems [1]. In the light of soft systems concept, all relevant parties and their roles can be clearly identified. The problem should also be expressed in a way to include the objectives, needs, resources and constraints.

The second important issue is the iterative process in knowledge acquisition. All existing approaches can be regarded as iterative processes which aim at verifying the correctness of the knowledge base and validating the adequacy of the implemented system. However, there is no guidance provided for measure of performance of each stage of the whole knowledge acquisition process. The feedback decisions would be based on the arbitrary experience and judgement of an individual.

To overcome the above problems, a methodology for establishing the expert system development system (ESDS) has been introduced. This approach considers the expert system develop- 


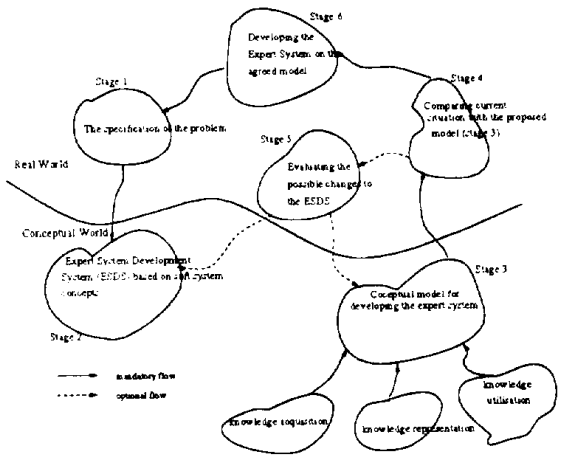

Figure 2: The methodology for establishing ESDS

ment environment as a soft system which can be characterised by the following components:

1. Objective: The ESDS aims at developing expert systems which can satisfy requirements of relevant parties and the environmental constraints. More important, the ESDS should have the capacity to handle uncertainty and changes.

2. Components The components of the ESDS can be divided into three categories: human resources, computing resources, and processed elements. Human resources include software developer, domain experts, users and the system owner. Computing resources include software tools and hardware equipment. Processed elements are those related to human knowledge, information and decisions.

3. Connectivity: The components which make up the ESDS form a degree of connectivity. They are more closely connected to each other than the components outside the ESDS. The connectivity indicates the information flow and operation sequence among different system components.

4. Subsystems: The subsystem of the ESDS may be defined according to the main functions of the whole development process. Some commonly accepted subsystems of the ESDS include knowledge engineering subsystem, system development subsystem, and system acceptance subsystem. The connectivity of the system implies that the subsystems may have boundaries, across which will be flows of information, and perhaps resources. A more important property is that the activities of the system are activated within the boundaries of the subsystems.

5. Wider System: The ESDS itself may be a subsystem of another system which can be regarded as a wider system. The destination of wider system of the ESDS depends on the issue that the ESDS is concerned. For instance, if the main concern of establishing the ESDS is to improve the effectiveness of the overall computerisation of the organisation, the wider system of the ESDS would be the business information system of the organisation.

6. Measures of Performance: In the ESDS, it is important to determine whether the employed approach is heading a correct direction. Measures of performance should be set for evaluating the effectiveness of some activities and the values of some relevant entities and their relationships in the ESDS. A good use of the measure of performance would be able to help us to cope with the ever changing situation in most real life problems.

The methodology for establishing the ESDS comprises six stages as shown in Figure 2. Stages $1,4,5$ and 6 are concerned with real world situation, and stages 2 and 3 are related to the conceptual world. Stage 1 intends to understand the situation by describing the present problem which currently has not been attempted by a knowledge based system. In stage 2, the ESDS will be defined with respect to the central issue of the problem situation. We may look ahead into stages 4 and 5 to polish the definition such that it can help to reveal problems within the ESDS. Generally speaking, the ESDS aims at building expert system through knowledge engineering. This can be considered as a transformation process. Stage 3 aims at constructing a conceptual model which consists of a minimum number of necessary activities for achieving the transformation described by the ESDS. To determine the practicality of the conceptual 
model, it is compared with the real life situation or existing approach in stage 4 . These changes may cause re-arrangement in the structure and procedures of the ESDS. Stage 6 represents the actual implementation phase of the expert system based on the agreed conceptual model. This methodology forms a cycle which allows improvement to be made on the ESDS.

\section{Conclusion}

Although a great deal of research has been published in the context of expert system development, very little attention has been given to the efficiency and effectiveness of the development process. In order to examine all relevant facets in the process of knowledge engineering, the soft systems concept is introduced. Applying the soft systems concept on expert systems development can be a useful way of thinking about the overall situation of the development and maintenance of the expert system. It provides a framework for visualising internal and external factors as an integrated entity. This concept also allows recognition of the proper place and functions of all associates. Through the soft systems concept, the major problems that may occur in the development life cycle can be encountered by the relevant parties. The methodology introduced in this paper can be regarded as a strategic model for expert system development while other existing knowledge-based system development methodologies are considered as tactical approaches. In other word, the soft systems approach help establishing the development environment and the technical methodologies. These two kinds of methodologies should be complementing each other and $\mathrm{i}$ co-exist to overcome both soft and hard problems. The success of a knowledge-based system development depends on the determination of measures of performance for individual development processes. However, a limited effort has been contributed to measures of performance for the ESDS. We are currently investigating this issue, so that the control mechanisms for evaluating the effectiveness of the ESDS can be established.

\section{References}

[1] A. H. Abdul-Gader. Usability of knowledgebased systems: Beyond technical feasibility. Information $\mathcal{E}$ Management, 21:1-6, 1991.

[2] B. G. Buchanan and E. H. Shortliffe. RuleBased Expert Systems. Addison Wesley, Reading, MA, 1984.

[3] P. B. Checkland. Systems Thinking, Systems Practice. John Wiley \& Sons, Chichester, 1981.

[4] Cheong D.K., Strobel J., Biswas G., Lee G., Kendall C.G., Cannon R., and Bezdek J. Playmaker, a knowledge based expert system. Geobyte, 7(6):28-41, 1993.

[5] B. Gaines. An overview of knowledgeacquisition and transfer. Journal of ManMachine Studies, (26):453-472, 1987.

[6] M. R. Genesereth. The use of design descriptions in automated diagnosis. Artificial Intelligence, 24:411-436, 1984.

[7] P. Jackson. Introduction to Expert Systems. Addison-Wesley, 1990.

[8] A. Kraft. XCON: An expert system configuration system at Digital Equipment Corporation. MIT-Press, 1984.

[9] J. Martin and S. Oxman. Prentice Hall, 1988.

[10] J. R. Olson and H. H. Rueter. Extracting expertise from experts: Methods for knowledge acquisition. Expert Systems, 4(3):152$168,1987$.

[11] Duda R.O., Hart P.E., Barrett P., Gaschnig J., K. Konolige, Reboh R., and Slocum J. Development of the prospector system for mineral exploration. Final report, projects 5821 and 6415, SRI International, Menlo Park, California, 1978. 